Präv Gesundheitsf 2019 · 14:398-402 https://doi.org/10.1007/s11553-018-0694-6

Eingegangen: 5. Oktober 2018

Angenommen: 4. Dezember 2018

Online publiziert: 12. Februar 2019

(c) Der/die Autor(en) 2018

CrossMark

\author{
B. Biallas ${ }^{1,3}\left(\mathbb{D} \cdot\right.$ D. Schäfer ${ }^{1}$ L. Dejonghe ${ }^{1} \cdot$ L. Franz $^{1} \cdot$ K. Petrowski $^{2} \cdot$ I. Froböse ${ }^{1}$. \\ C. Wilke ${ }^{1}$ \\ ${ }^{1}$ Institut für Bewegungstherapie und bewegungsorientierte Prävention und Rehabilitation, Deutsche \\ Sporthochschule Köln, Köln, Deutschland \\ ${ }^{2}$ Medizinische Psychologie und Medizinische Soziologie, Johannes-Gutenberg Universität Mainz, Mainz, \\ Deutschland \\ ${ }^{3}$ Zentrum für Gesundheit durch Sport und Bewegung, Deutsche Sporthochschule Köln, Köln, Deutschland
}

\title{
Präventionsreife in kleinen und mittleren Unternehmen
}

\section{Analyse hinsichtlich Präventionswillen, Präventionsinfrastruktur und des Umsetzungsgrads gesundheitsförderlicher Maßnahmen}

Einigen Großunternehmen ist die Bedeutung von betrieblicher Gesundheitsförderung (BGF) bereits bewusst, sodass sie vermehrt BGF umsetzen. Kleine und mittlere Unternehmen (KMU) reagieren nach wie vor zurückhaltender. Aufgrund ihrer strukturellen Besonderheiten stellen KMU eine eigenständige Zielgruppe der BGF dar, der 99,3\% der deutschen Unternehmen zugehören [12]. Es zeigt sich der Bedarf an angepassten Konzepten zur Umsetzung von BGF in KMU. Vor diesem Hintergrund analysiert die vorliegende Studie KMU hinsichtlich der Präventionsreife und des Umsetzungsgrades gesundheitsfördernder Maßnahmen.

\section{Hintergrund und Fragestellung}

Unter dem Begriff der BGF werden sämtliche von Arbeitgebern, Arbeitnehmern oder der Gesellschaft durchgeführte Maßnahmen zur Verbesserung von Gesundheit und Wohlbefinden am Arbeitsplatz zusammengefasst. Eine systematische Einführung und Umsetzung von BGF kann neben der Steigerung des Wohlbefindens und der Leistungsfähigkeit der Mitarbeiter auch die Produktivität des Unternehmens verbessern [3].

Studien zeigen eine Abhängigkeit des Angebots gesundheitsfördernder Maß- nahmen von der Betriebsgröße, wobei die Häufigkeit der BGF-Aktivität mit der Unternehmensgröße steigt [11]. Unternehmen mit weniger als 499 Mitarbeitern oder einem Jahresumsatz von maximal 50 Mio. $€$ zählen zur Gruppe der KMU [6]. Sie werden mit ähnlichen Herausforderungen wie große Konzerne konfrontiert, da demographische Entwicklung, Arbeitsverdichtung, technischer Fortschritt oder der absehbare Mangel an Fachkräften den Arbeitsmarkt gleichermaßen prägen $[8,9]$. Jedoch weisen KMU andere personelle und organisatorische Strukturen auf und sind als eigenständige Zielgruppe der BGF zu betrachten, auf die sich bestehende Konzepte für Großunternehmen nicht direkt übertragen lassen. Dies ist eine mögliche Erklärung, warum in KMU BGF-Maßnahmen noch deutlich weniger verbreitet sind. Im Hinblick darauf, dass 60,2 \% der Arbeitnehmer in KMU beschäftigt sind, wird die Relevanz der Entwicklung von angepassten BGF-Konzepten für KMU deutlich [12].

Hierbei erweist sich die Präventionsreife als geeignetes Konzept. Es bildet den Grad des Willens und die Fähigkeit eines sozialen Systems ab, Gesundheitsförderung und Prävention systematisch und dauerhaft zu betreiben $[7,10]$. Dabei wird zwischen den Dimensionen
Präventionswille und Präventionsinfrastruktur unterschieden. Der Präventionswille misst den Willen eines Sozialsystems, BGF-Maßnahmen zu planen, einzuleiten und durchzuführen. Die Präventionsinfrastruktur beschreibt die Fähigkeit eines sozialen Systems, BGFMaßnahmen über gegebene materielle und immaterielle Ressourcen, sowie institutionalisierte Prozesse eines Betriebs durchzuführen [7, 10]. Anhand dieser Dimensionen lassen sich Unternehmen einer von vier Präventionsstufen zuordnen, die in $\bullet$ Tab. 1 modifiziert nach Jung et al. [7] dargestellt sind.

Je höher die Präventionsreife, desto besser kann ein soziales System präventive und gesundheitsfördernde Maßnahmen durchführen. Demnach ist die Implementierung von BGF in sozialen Systemen abhängig von der Präventionsreife $[7,10]$. Die Präventionsreife wird von verschiedenen BGF-Merkmalen eines Unternehmens beeinflusst. Größe, Informationsstand, bestehende BGFMaßnahmen und Umsetzbarkeit wirken sich auf die Präventionsreife eines Unternehmens aus [7, 11]. KMU zeigen nicht nur in Bezug auf die Unternehmensgröße Besonderheiten in diesen Merkmalen. Dies ist bei der Erstellung von BGF-Konzepten zu beachten. 
Tab. 1 Stufen der Präventionsreife [7]

\begin{tabular}{l|lll}
\hline & \multicolumn{2}{l}{ Präventionsinfrastruktur } & \\
& & Vorhanden & Nicht vorhanden \\
\hline $\begin{array}{l}\text { Präventions- } \\
\text { wille }\end{array}$ & Vorhanden & $\begin{array}{l}\text { Grad der Präventionsreife: } \\
\text { hoch } \\
\text { (Präventionsstufe A) }\end{array}$ & $\begin{array}{l}\text { Grad der Präventionsreife: } \\
\text { mittel } \\
\text { (Präventionsstufe B) }\end{array}$ \\
\cline { 2 - 4 } & Nicht vorhanden & $\begin{array}{l}\text { Grad der Präventionsreife: } \\
\text { mittel } \\
\text { (Präventionsstufe C) }\end{array}$ & $\begin{array}{l}\text { Grad der Präventionsreife: } \\
\text { gering }\end{array}$ \\
\hline
\end{tabular}

In einer Studie von Schaefer et al. [11] erweisen sich „fehlende finanzielle und personelle Ressourcen“, „Priorität für andere Themen“ sowie „fehlende Fachkenntnisse“ als entscheidende Hindernisse für die Einführung von BGF in KMU. Über $40 \%$ der befragten Unternehmen geben an „noch nicht über BGF nachgedacht“ $\mathrm{zu}$ haben [11]. Zudem ist die Realisierbarkeit der BGF in KMU stark von der Einstellung, Motivation, Lebensweise und dem Führungsstil des Geschäftsführers abhängig, da er alleine die Betriebskultur und das Führungsverhalten dominiert $[8,9]$. Potenziale für BGF bestehen in der flachen personenbezogenen Hierarchie, einer geringen Formalisierung, guten sozialen Beziehungen, einer hohen Autonomie der Mitarbeiter sowie kurzen, direkten Informations- und Kommunikationswegen [8].

Vor diesem Hintergrund betrachtet die vorliegende Arbeit den Umsetzungsgrad und die Präventionsreife gesundheitsfördernder Maßnahmen von KMU des TechnologieParks Köln. Im Fokus der Arbeit stehen die Bedürfnisse und Haltungen der KMU zur BGF sowie deren grundlegende Möglichkeiten und Hindernisse in Bezug auf die Umsetzung von BGF. Es ergeben sich folgende zentrale Fragestellungen:

- Inwieweit sind KMU mit gesundheitsfördernden Maßnahmen vertraut und setzen diese um?

- Welche Zusammenhänge bestehen zwischen der Präventionsreife und der Umsetzung von BGF-Maßnahmen in KMU?

\section{Studiendesign und Untersuchungsmethoden}

Im Rahmen der Querschnittsstudie wurden KMU (1-499 Mitarbeiter) des Tech-
nologieParks Köln hinsichtlich ihrer Präventionsreife und des Umsetzungsgrades von BGF-Maßnahmen analysiert. Als Erhebungsinstrument diente ein Fragebogen, der zusammen mit einem persönlichen Anschreiben in ausgedruckter Form mittels vorfrankierter Rücksendeumschläge an 90 KMU des TechnologieParks ausgehändigt wurde. Pro Unternehmen sollte jeweils eine Person den Fragebogen als Stellvertreter innerhalb von 2 Wochen beantworten. Insgesamt erstreckte sich die Erhebung über 4 Wochen.

Der Fragebogen gliederte sich in einen standardisierten und einen nichtstandardisierten Teil. Einige allgemeine Fragen zielten auf die Erhebung sozialdemographischer Daten der stellvertretenden Person des Unternehmens ab. Zur Erfassung der Präventionsreife diente das „worksite health promotion capacity instrument" (WHPCI), ein zweidimensionales Messinstrument mit intervallskalierten Likert-Antworten (von $0=$ „stimme überhaupt nicht $\mathrm{zu}$ “ bis $10=$ „stimme voll und ganz zu“; [7]). Insgesamt wurden 12 Items zur quantitativen Auswertung herangezogen und den beiden Subskalen „Präventionswille“ und „Präventionsinfrastruktur“ zugeordnet. Zur Beurteilung des Umsetzungsgrades gesundheitsfördernder Maßnahmen in den Unternehmen wurden offene und geschlossene Fragen in Anlehnung an den „Fragebogen zur Betrieblichen Gesundheitsförderung “von Hirtenlehner und Sebinger (2004) konzipiert [4]. Weitere Fragen dienten der Erfassung von Potenzialen und Problematiken in Bezug auf die Implementation von BGF in KMU. Dabei bezogen sich Fragen zu Bedürfnissen und Haltungen der KMU zur BGF auf den Präventionswillen. Fragen zu gegebenen Möglichkeiten und Hindernisse bei der
Umsetzung von BGF bezogen sich auf die Präventionsinfrastruktur.

Die Auswertung der Daten erfolgte über das Datenanalyseprogramm IBM SPSS Statistics 22 und das Tabellenkalkulationsprogramm Microsoft Excel 2010. Dabei kamen sowohl Verfahren der deskriptiven als auch der analytischen Statistik zum Einsatz. Ab einer Irrtumswahrscheinlichkeit von $p \leq 0,05$ galt das Ergebnis als signifikant [2]. Für die deskriptive Analyse der Präventionsreife und des Umsetzungsgrades gesundheitsfördernder Maßnahmen wurden Häufigkeiten, Mittelwerte (M) und Standardabweichungen (SD) berechnet. Die Kategorisierung der KMU hinsichtlich des Grades der Präventionsreife erfolgte durch die Bestimmung von Cut-offWerten mithilfe des Youden-Index. Der Youden-Index Y = 1 wurde für den Cutoff-Wert von 6 berechnet. Alle Unternehmen mit einem Durchschnittswert von $<6$ wurden der Kategorie „Präventionswille nicht vorhanden“ zugeordnet, während alle anderen als „Präventionswille vorhanden“ eingestuft wurden [7]. Die Dimension Präventionsinfrastruktur wurde durch einen Cut-off-Wert von 2,55 bei einem Youden-Index von $\mathrm{Y}=1$ gekennzeichnet. Unternehmen mit einem Cut-off-Wert von $<2,55$ wurden der Kategorie „Präventionsinfrastruktur nicht vorhanden“ bzw. alle anderen der Kategorie „Präventionsinfrastruktur vorhanden" zugeordnet [7]. Des Weiteren wurde anhand einer Korrelationsanalyse der Zusammenhang zwischen den Dimensionen „Präventionswillen“ und „Präventionsinfrastruktur" mit unterschiedlichen Variablen überprüft. Für nicht normalverteilte Variablen und bei nur einer normalverteilten Variablen wurden die Korrelationen mittels der Spearman-Korrelationsanalyse ermittelt. Bei zwei normalverteilten Variablen wurde die Bravais-Pearson-Korrelationsanalyse durchgeführt. Anhand des Korrelationskoeffizienten $1 \leq \mathrm{r} \geq-1$ konnten Aussagen über die Stärke und die Richtung des Zusammenhangs getroffen werden. Ab 0,5 $<\mathrm{r} \leq 0,7$ wurde von einer mittleren Korrelation ausgegangen [2]. Zur Überprüfung der Daten auf Unterschiede zwischen der Präventionsreife und der Durchführung von BGF-Maß- 
Präv Gesundheitsf 2019 • 14:398-402 https://doi.org/10.1007/s11553-018-0694-6

(c) Der/die Autor(en) 2018

B. Biallas · D. Schäfer · L. Dejonghe · L. Franz · K. Petrowski · I. Froböse · C. Wilke

\section{Präventionsreife in kleinen und mittleren Unternehmen. Analyse hinsichtlich Präventionswillen, Präventionsinfrastruktur und des Umsetzungsgrads gesundheitsförderlicher Maßnahmen}

\section{Zusammenfassung}

Hintergrund. Großunternehmen haben die zunehmende Relevanz von betrieblicher Gesundheitsförderung (BGF) erkannt und Maßnahmen integriert. Kleine und mittlere Unternehmen (KMU) reagieren zurückhaltender.

Ziel der Arbeit. Das Ziel der vorliegenden Studie liegt in der Erfassung der Präventionsreife und des Umsetzungsgrades von BGF in KMU. Methodik. In Form einer Querschnittsstudie wurden KMU $(n=36)$ des TechnologieParks Köln untersucht. Pro Unternehmen füllte ein Arbeitnehmer stellvertretend für alle Angestellten einen Fragebogen aus. Dabei spielte die berufliche Stellung der Person keine Rolle.
Der Fragebogen erfasste Präventionsreife, den Umsetzungsgrad von BGF in KMU und BGF-Merkmale. In der Auswertung wurde der Zusammenhang der BGF-Merkmale mit der Präventionsreife betrachtet.

Ergebnisse. Etwa 50\% der befragten KMU setzen BGF um. $13 \mathrm{KMU}$ weisen eine hohe, hingegen 18 eine geringe Präventionsreife auf. Der Präventionswillen ist im Mittel gering (Mittelwert $[\mathrm{M}] \pm$ Standardabweichung $[\mathrm{SD}]=$ $5,07 \pm 3,23)$. Hingegen wird der Cut-off-Wert für eine vorhandene Präventionsinfrastruktur im Mittel erreicht $(M \pm S D=2,69 \pm 2,76)$. Unternehmensgröße, Informationsstand, Durchführung und Umsetzbarkeit von
BGF-Maßnahmen korrelieren signifikant $(p \leq 0,05)$ mit Präventionswillen und Präventionsinfrastruktur.

Diskussion. KMU stellen eine wichtige Zielgruppe für BGF-Maßnahmen dar. Durch gezielte Maßnahmen wie eine verstärkte Informationspolitik, Ressourcenstärkung und KMU-Netzwerken kann die Präventionsreife von KMU gestärkt und der Umsetzungsgrad von BGF-Maßnahmen erhöht werden.

\section{Schlüsselwörter}

Betriebliche Gesundheitsförderung · Betriebliches Gesundheitsmanagement · Prävention · Präventionswille · Präventionsinfrastruktur

\section{Prevention culture in small and medium-sized companies. Analysis in terms of health promotion willingness, health promotion management and the degree of implementation of health promotion measures}

\section{Abstract}

Background. Small and medium-sized enterprises (SME) are practicing less worksite health promotion (WHP) than large companies. Objectives. The aim of the study was to analyse the health promotion capacity and the implementation level of health promotion activities in SME in the TechnologiePark Cologne.

Methods. A questionnaire, which was answered by one person per company, served for collecting data for this cross-sectional study. SMEs $(n=36)$ of the TechnologyPark Cologne were asked for the health promotion willingness, health promotion management, needs and attitudes of the enterprises to WHP, as well as their possibilities and limitations with regard to implementation. In addition, the present study assesses the relationship between health promotion capacity and different characteristics of WHP.

Results. Half of the companies implemented activities for WHP. In all, 13 SME have a high, but 18 a very low level of health promotion capacity. On average the health promotion willingness of SMEs is low (mean $[\mathrm{M}] \pm$ standard deviation $[S D]=5.07 \pm 3.23$ ). Conversely the health promotion management seems to be better $(M \pm S D=2.69 \pm 2.76)$. The characteristics, company size, level of information, feasibility and implementation of WHP activities correlate significantly $(p \leq 0.05)$ with health promotion willingness and health promotion management.

Conclusion. SMEs are an important target group for WHP activities because of their economic and health policy implications. Specific measures like strengthening information policy, improvement of resources and SME networks could enhance the health promotion capacity of SMEs and increase the implementation of WHP in SMEs.

Keywords

Workplace health promotion $\cdot$ Health promotion management · Prevention · Health promotion willingness $\cdot$ Health promotion capacity nahmen wurde der Mann-Whitney-UTest durchgeführt.

\section{Ergebnisse}

Mit einer Rücklaufquote von $40 \%$ ergab sich eine Gesamtstichprobe von 36 KMU. Diese unterteilt sich nach KMU-Definition des Instituts für Mittelstandsforschung Bonn in 14 Kleinstbetriebe, 12 Kleinbetriebe und 10 mittlere Betriebe [9].
Die Auswertung der Fragebögen zeigt einen Umsetzungsgrad von $50 \%$. Demnach hat die Hälfte der untersuchten Unternehmen bereits BGF-Maßnahmen implementiert ( $50 \%, n=18)$. Dabei zeigt sich, dass mittlere Unternehmen (80\%) und Kleinunternehmen $(66,7 \%)$ zu einem größeren Anteil BGF durchführen als Kleinstunternehmen (14,3\%).

Zur Beurteilung der Präventionsreife der befragten KMU erfolgt eine Einteilung in die vier Präventionsreifestufen nach Jung et al. [7]. $13 \mathrm{KMU}(36,1 \%)$ sind der Präventionsstufe A („hohe Präventionsreife") zuzuordnen, da sowohl Präventionswille als auch Präventionsinfrastruktur im Unternehmen gegeben sind. Drei Unternehmen $(8,3 \%)$ sind Stufe B („mittlere Präventionsreife“) zuzuordnen, da nur der Präventionswille vorhanden ist. Zwei Unternehmen (5,5\%) verfügen lediglich über eine Präventionsinfrastruktur und gehören damit zu Stufe C („mittlere Präventionsreife“). Die Mehrheit der Unternehmen (50\%, $n=18$ ) verfügt weder über Präventions- 
Tab. 2 Korrelationsanalyse zwischen den BGF-Merkmalen und den Dimensionen Präventionswille und Präventionsinfrastruktur

\begin{tabular}{lllll} 
& \multicolumn{2}{l}{ Präventionswille } & \multicolumn{2}{l}{ Präventionsinfrastruktur } \\
& r & p & r & p \\
\hline Durchführung von BGF & $-0,720$ & $\leq 0,001$ & $-0,757$ & $\leq 0,001$ \\
Unternehmensgröße & 0,355 & $\leq 0,05$ & 0,363 & $\leq 0,05$ \\
Umsetzbarkeit & $-0,547$ & $\leq 0,001$ & $-0,529$ & $\leq 0,001$ \\
\hline $\begin{array}{l}\text { Informationsstand } \\
\text { Themen und Inhalte }\end{array}$ & $-0,576$ & $\leq 0,001$ & $-0,602$ & $\leq 0,001$ \\
\hline Methoden und Ablauf & $-0,577$ & $\leq 0,001$ & $-0,614$ & $\leq 0,001$ \\
\hline Nutzen und Wirkung & $-0,671$ & $\leq 0,001$ & $-0,577$ & $\leq 0,001$ \\
\hline Anbieter und Anlaufstelle & $-0,701$ & $\leq 0,001$ & $-0,648$ & $\leq 0,001$
\end{tabular}

wille noch über Präventionsreife und ist somit der Stufe D (,geringe Präventionsreife") zuzuordnen. Die Dimensionen der Präventionsreife sind auf einer Skala von 1 („stimme überhaupt nicht $\left.\mathrm{zu}^{\prime \prime}\right)$ bis 10 („stimme voll und ganz $\mathrm{zu}^{\text {“) }}$ erfasst. Der Mittelwert für den Präventionswillen aller Betriebe liegt bei $M \pm S D=5,07 \pm 3,23$. Damit wird der Cut-off-Wert von $\geq 6$ für einen vorhandenen Präventionswillen im Mittel nicht erreicht. Für die Präventionsinfrastruktur der Betriebe zeigt sich ein geringerer Wert von $\mathrm{M} \pm \mathrm{SD}=2,69 \pm 2,76$. Damit wird der Cut-off-Wert für eine vorhandene Präventionsinfrastruktur von $\geq 2,55$ im Mittel erreicht. Es ergeben sich signifikante Korrelationen der Unternehmensmerkmale Unternehmensgröße, Informationsstand, Durchführung und Umsetzbarkeit von BGF-Maßnahmen mit dem Präventionswillen und der Präventionsinfrastruktur, die in • Tab. 2 ersichtlich werden.

\section{Diskussion}

Laut Ergebnis setzen $50 \%$ der befragten KMU bereits BGF-Maßnahmen um. Weitere Studien ermitteln geringere Anteile $[4,5]$. Zwar ist ein direkter Vergleich mit diesen Studien schwierig, da häufig eine andere Definition der KMU verwendet wird, jedoch wird die geringe Verbreitung von BGF in KMU deutlich. Bei Betrachtung der Präventionsreife der untersuchten KMU zeigt sich, dass die Mehrheit (50 \%) nur „gering präventionsreif “ ist. Anhand der Mittelwerte lässt sich darauf schließen, dass die Unternehmen im Durchschnitt über ei- ne gute Präventionsinfrastruktur verfügen. Der Präventionswille ist hingegen in vielen KMU zu gering. Sowohl Präventionswille, als auch Präventionsinfrastruktur korrelieren mit allen BGF-Merkmalen (• Tab. 2). Folglich scheint ein $\mathrm{Zu}$ sammenhang zwischen Größe, Umsetzbarkeit und Informationsstand eines Unternehmens und seiner Präventionsreife zu bestehen. Es zeigt sich die Tendenz, dass KMU mit einem besseren Präventionswillen oder einer besseren Präventionsreife auch zu einem höheren Anteil BGF-Maßnahmen durchführen [6]. Anhand erfragter Bedürfnisse, Haltungen, grundlegender Möglichkeiten und Hindernissen lassen sich Potenziale und Problematiken der KMU im Rahmen der Umsetzung von BGF durch eine Verbesserung der Präventionsreife ableiten.

Das größte Potenzial von BGF sehen befragte KMU in der Steigerung des Gesundheitsbewusstseins und des Gesundheitszustands. Mit einer Produktivitätssteigerung oder verbesserten Arbeitsabläufen rechnen wenige. Bei der Überprüfung des Wissensstands der KMU zu BGF wird ein Informationsmangel deutlich. Dieser besteht v. a. in den Bereichen „methodisches Vorgehen und Ablauf“ sowie „potenzielle Anbieter und Anlaufstellen“. In der Befragung nennen KMU den Informationsmangel neben Ressourcenmangel und fehlendem Interesse als Hindernis für die Einführung von BGF in KMU. Die häufigsten Hindernisse scheinen jedoch der „Vorrang des Tagesgeschäfts“ und der „Vorrang anderer Themen“ zu sein $[4,9,11]$. Zudem erwarten nur 5,6\% keine Schwierigkeiten bei der Einführung von BGF in KMU. Folg- lich sind gezielte Maßnahmen notwendig, um den Mangel an Informationen, das fehlende Bewusstsein sowie fehlende finanzielle und personelle Ressourcen als entscheidende Ursachen für die geringe Umsetzung von BGF in KMU zu reduzieren [1, 3].

Ein möglicher Lösungsansatz besteht in einer gesteigerten Informationspolitik. Medien, Vorträge oder Expertengespräche helfen Informationsstand und Bewusstsein zu verbessern. Neben Basiswissen zu BGF sollten dabei v.a. Informationen zu positiven Auswirkungen von BGF auf die Leistung, Motivation und Gesundheit der Mitarbeiter gegeben werden. Dabei gilt es, Bedürfnisse und Wünsche der Mitarbeiter zu berücksichtigen. Zudem sind weitere Vorteile für das Unternehmen und Informationen zu Anbietern und Anlaufstellen zu vermitteln [1]. Wichtig ist dabei, dass Informationen und Angebote die richtigen Ansprechpartner des Unternehmens erreichen. Aus der Befragung von KMU, die bereits BGF durchführen, geht hervor, dass v. a. Unternehmensleitung und Personalleiter zu informieren sind, da sie am häufigsten die Initiative für BGF ergreifen [1]. Hilfreich wäre auch ein Austausch der Informationen verschiedener KMU untereinander. Dies gelingt durch einen Zusammenschluss mehrerer Unternehmen zu einem KMU-Netzwerk. Durch die gegenseitige Unterstützung innerhalb solcher Netzwerke können bei geringem Aufwand der einzelnen Betriebe gemeinsame Maßnahmen und umfassende Lösungen zur Überwindung von mangelnden Ressourcen entwickelt werden [11]. Zudem sind Gesundheitsträger eher bereit KMU-Netzwerke zu unterstützen, da diese über eine höhere Reichweite und eine höhere Arbeitnehmeranzahl verfügen als einzelne KMU [5].

Neben den genannten Ansätzen zur Stärkung der Präventionsreife sollten BGF-Konzepte auch strukturelle Vorteile von KMU berücksichtigen. So ermöglicht die geringere Beschäftigtenanzahl eine direktere und persönlichere Kommunikation. Maßnahmen können gezielter auf individuelle Vorstellungen und Bedürfnisse der Mitarbeiter angepasst werden. 


\section{Limitationen}

Beim Untersuchungsgut handelt es sich um eine kleine Stichprobe aus KMU des TechologieParks Köln. Die vorliegenden Ergebnisse lassen somit keine allgemeingültigen Aussagen zu. Darüber hinaus sollten in zukünftigen Studien der Einfluss und die Perspektiven verschiedener Interessensgruppen beim Ausfüllen des Fragebogens vermehrt Berücksichtigung finden.

\section{Fazit für die Praxis}

- Nur $50 \%$ der KMU setzen bereits BGF-Maßnahmen um. Damit wird die geringe Verbreitung von BGF in KMU deutlich.

- Das Konstrukt der Präventionsreife bietet einen geeigneten Ansatz, um strukturelle Besonderheiten in Bezug auf die Umsetzung von BGF in KMU aufzudecken. So ist eine niedrige Präventionsreife eine mögliche Ursache für die geringe Verbreitung von BGF in KMU.

- Untersuchungen zeigen einen Zusammenhang zwischen Unternehmensmerkmalen und den Präventionsreifedimensionen Präventionswille und Präventionsinfrastruktur. Dabei werden v. a. fehlendes Bewusstsein sowie Informations- und Ressourcenmangel als zentrale Hindernisse bei der Umsetzung von BGF in KMU deutlich. Folglich gilt es, passende BGF-Konzepte zu entwickeln, die entsprechende Maßnahmen beinhalten. So können eine verstärkte Informationspolitik und der Zusammenschluss einzelner KMU zu KMUNetzwerken die Präventionsreife der KMU und dadurch die Verbreitung von BGF in KMU erhöhen.

\section{Korrespondenzadresse

\begin{tabular}{l} 
Dr. B. Biallas \\
$\begin{array}{l}\text { Zentrum für Gesundheit } \\
\text { durch Sport und Bewegung, } \\
\text { Deutsche Sporthochschule } \\
\text { Köln } \\
\text { Köln, Deutschland } \\
\text { biallas@dshs-koeln.de }\end{array}$ \\
\hline
\end{tabular}

Einhaltung ethischer Richtlinien

Interessenkonflikt. B. Biallas, D. Schäfer, L. Dejonghe, L. Franz, K. Petrowski, I. Froböse und C. Wilke geben an, dass kein Interessenkonflikt besteht.

Alle beschriebenen Untersuchungen am Menschen wurden mit Zustimmung der zuständigen Ethik-Kommission, im Einklang mit nationalem Recht sowie gemäß der Deklaration von Helsinki von 1975 (in der aktuellen, überarbeiteten Fassung) durchgeführt. Von allen beteiligten Patienten liegt eine Einverständniserklärung vor.

Open Access Dieser Artikel wird unter der Creative Commons Namensnennung 4.0 International Lizenz (http://creativecommons.org/licenses/by/4.0/deed. de) veröffentlicht, welche die Nutzung, Vervielfältigung, Bearbeitung, Verbreitung und Wiedergabe in jeglichem Medium und Format erlaubt, sofern Sie den/die ursprünglichen Autor(en) und die Quelle ordnungsgemäßnennen, einen Linkzur Creative Commons Lizenz beifügen und angeben, ob Änderungen vorgenommen wurden.

\section{Literatur}

1. Bechmann S, Jäckle $R$, Lück $P$ (2011) iga-Report 20: Motive und Hemmnisse für Betriebliches Gesundheitsmanagement (BGM). Umfrage und Empfehlungen. AOK-Bundesverband, Berlin, Essen

2. Bühl A (2012) SPSS 20. Einführung in die moderne Datenanalyse, 13. Aufl. Always learning. Pearson, München

3. Europäisches Netzwerk für Betriebliche Gesundheitsförderung (2014) Luxemburger Deklaration zur betrieblichen Gesundheitsförderung in der Europäischen Union. BKK Bundesverband, Luxemburg

4. Hirtenlehner $H$, Sebinger S (2004) Betriebliche Gesundheitsförderung für KMU. Ergebnisse einer Bedarfsanalyse für Oberösterreich. OÖ GKK, Institut für Gesundheitsplanung, Linz

5. Hollederer A (2007) Betriebliche Gesundheitsförderung in Deutschland. Ergebnisse des IABBetriebspanels 2002 und 2004. Gesundheitswesen 69(2):63-76. https://doi.org/10.1055/s-2007970599

6. Ifm Bonn (2016) KMU-Definition des ifm Bonn. https://www.ifm-bonn.org/definitionen/kmudefinition-des-ifm-bonn/. Zugegriffen: 26. Sept. 2018

7. Jung J, Nitzsche A, Neumann M, Wirtz M, Kowalski C, Wasem J, Stieler-Lorenz B, Pfaff H (2010) The Worksite Health Promotion Capacity Instrument (WHPCI): development, validation and approaches for determining companies' levels of health promotion capacity. BMC Public Health 10:550. https://doi.org/10.1186/1471-2458-10-550

8. Meggeneder O (2017) ... zu teuer und zu aufwendig? Herausforderungen für die Betriebliche Gesundheitsförderung in Kleinen und Mittleren Unternehmen. In: Faller G, Abel B, Badura B, Bauer $G$ (Hrsg) Lehrbuch betriebliche Gesundheitsförderung, 3. Aufl. Hogrefe, Bern

9. Meyer JA (2008) Gesundheit in KMU: Widerstände gegen betriebliches Gesundheitsmanagement in kleinen und mittleren Unternehmen. Gründe, Bedingungen und Wege zur Überwindung. Techniker Krankenkasse, Hamburg
10. Pfaff $H$, Nitzsche A, Jung J (2004) Handbuch zum "Healthy Organisational Resources and Strategies" (HORST) Fragebogen. Veröffentlichungsreihe der Abteilung Medizinischen Soziologie des Instituts für Arbeitsmedizin, Sozialmedizin und Sozialhygiene der Universität zu Köln, Forschungsbericht 3/2008. Köln, Universität Köln

11. Schaefer E, Drexler H, Kiesel J (2016) Betriebliche Gesundheitsförderung in kleinen, mittleren und großen Unternehmen des Gesundheitssektors - Häufigkeit, Handlungsgründe der Unternehmensleitungen und Hürden der Realisierung. Gesundheitswesen 78(3):161-165. https://doi. org/10.1055/s-0034-1396887

12. Statistisches Bundesamt (2018) Anteile kleiner und mittlerer Unternehmen an ausgewählten Merkmalen. https://www.destatis. de/DE/ZahlenFakten/GesamtwirtschaftUmwelt/ UnternehmenHandwerk/KleineMittlereUnternehmen Mittelstand/Tabellen/Insgesamt.html. Zugegriffen: 26. Sept. 2018 